HNO 2022 $\cdot 70: 396-400$

https://doi.org/10.1007/s00106-021-01104-2

Angenommen: 14. Juli 2021

Online publiziert: 1. September 2021

(c) Der/die Autor(en) 2021

Redaktion

C. Matthias, Mainz

\section{Makroskopische und mikroskopische Veränderungen des N. vestibulocochlearis nach Gamma-Knife-Therapie}

\author{
Maximilian Scheer ${ }^{1}$. Christian Scheller ${ }^{1}$ Julian Prell ${ }^{1}$. Christian Mawrin ${ }^{2}$. \\ Torsten Rahne ${ }^{3}$. Christian Strauss ${ }^{1}$. Sebastian Simmermacher ${ }^{1}$ \\ ${ }^{1}$ Klinik für Neurochirurgie, Universitätsklinikum Halle, Medizinische Fakultät der Martin-Luther-Universität \\ Halle-Wittenberg, Halle, Deutschland \\ ${ }^{2}$ Institut für Neuropathologie, Universitätsklinikum Magdeburg, Magdeburg, Deutschland \\ ${ }^{3}$ Klinik für Hals-, Nasen-, Ohrenheilkunde, Universitätsklinikum Halle, Halle, Deutschland
}

\title{
Zusammenfassung
}

Wir berichten über einen Fall, bei dem makroskopische und mikroskopische Veränderungen des Verstibularnervs nach radiochirurgischer Behandlung eines intrameatalen Vestibularisschwannoms beobachtet wurden. Der Fallbericht zeigt das erste Mal ein morphologisches Korrelat der unerwünschten Effekte der GammaKnife-Therapie von Vestibularisschwannomen und unterstreicht, dass trotz eines deutlichen Abstands zum bestehenden Tumor degenerative Veränderungen der neuralen Strukturen erwartet werden können.

\section{Schlüsselwörter}

Radiochirurgie · Vestibularisschwannom · Komplikation · Demyelinisierung · Fallbericht

\section{Falldarstellung}

\section{Anamnese}

Bei einem 57-jährigen männlichen Patienten, welcher bis auf einen arteriellen Hypertonus gesund war, erfolgte drei Jahre vor der Konsultation in unserer Klinik eine Gamma-Knife-Therapie (13 Gy, Volumen $0,13 \mathrm{~cm}^{3}, 65 \%$ therapeutische Isodose, die Cochlea lag vollständig außerhalb der 6-Gy-Isodose) eines intrameatalen Vestibularisschwannoms (• Abb. 1). Vor der Gamma-Knife-Therapie beklagte der Patient einen progredienten Tinnitus sowie subjektiven Hörverlust über einen Zeitraum von knapp einem Jahr. Magnetresonanztomographie(MRT)-Verlaufskontrollen zeigten zunächst einen stabilen Befund. Nun berichtete der Patient von einem zunehmenden Tinnitus, einer Schwankschwindelsymptomatik, einer stetig zunehmenden Hörminderung, schmerzhaften Verkrampfungen der Ge- sichtsmuskulatur und einer Taubheit der linken Gesichtshälfte.

\section{Klinischer Befund}

Es bestand ein Hemispasmus facialis, eine Hypästhesie im Versorgungsgebiet des N. trigeminus (V2 und V3) und ein pathologischer Unterberger-Tretversuch sowie ein positiver Romberg-Test. Audiologisch zeigte sich eine hochgradige Einschränkung des Hörvermögens. Dieses hatte sich im Laufe der letzten 3 Jahre allmählich verschlechtert (- Abb. 2).

\section{Diagnose}

\section{Kranielle Bildgebung}

In der cMRT-Bildgebung zeigte sich im Vergleich zu den Voraufnahmen ein Progress des kontrastmittelaufnehmenden Tumoranteils im linken Kleinhirnbrückenwinkel. Ein Verlust der intrakapsulären Kontrastmittelaufnahme, wie es oft nach einer Ra- 

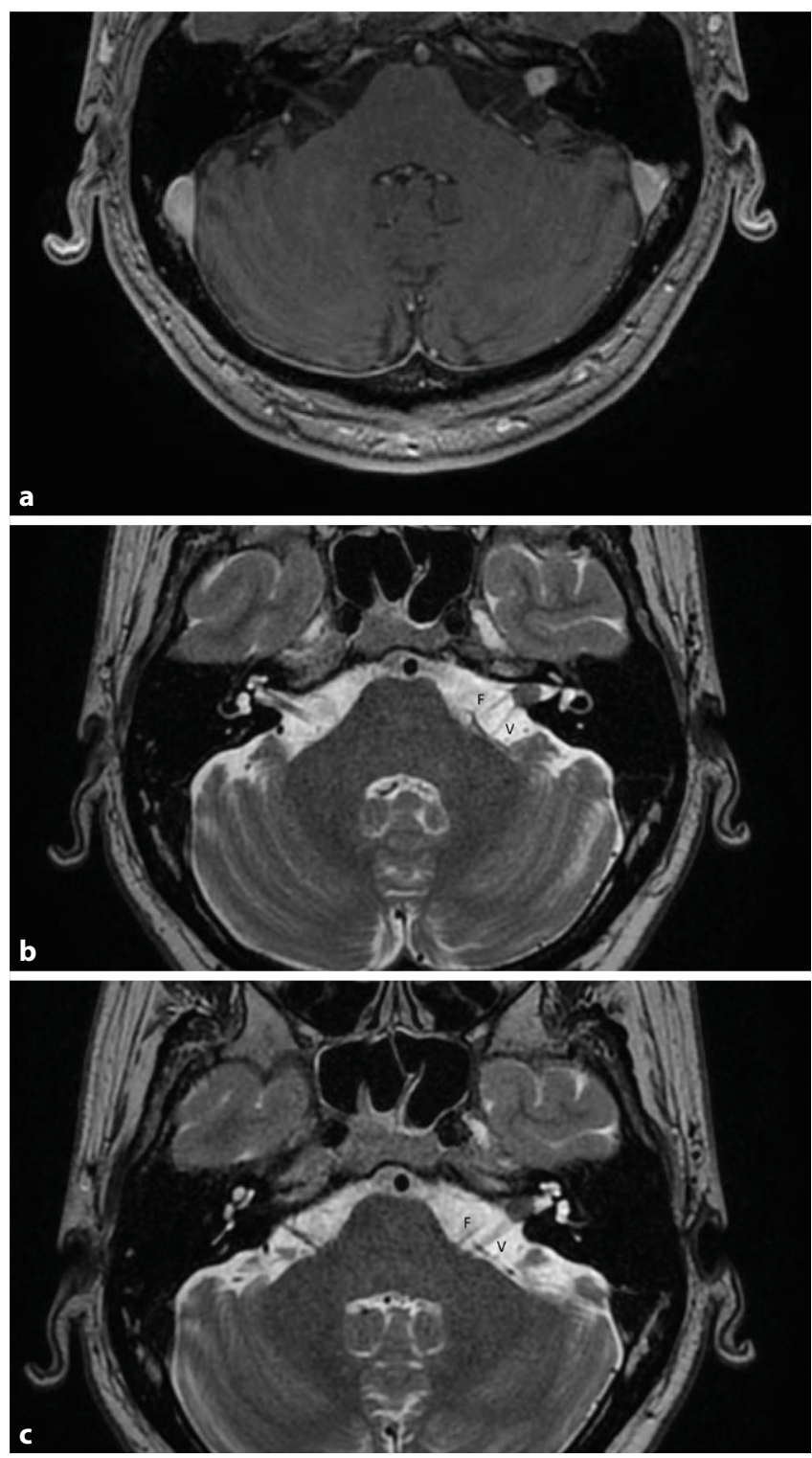

Abb. 1 4 cMRT im Jahr 2012; a T1 axial mit Kontrastmittel; Raumforderung im inneren Gehörgang, Indikation zur Gamma-Knife-Behandlung; $b$ und $\mathrm{C} T 2$ axial, Ausgangsbefund des N. facialis (F) und des N. vestibulocochlearis (V)

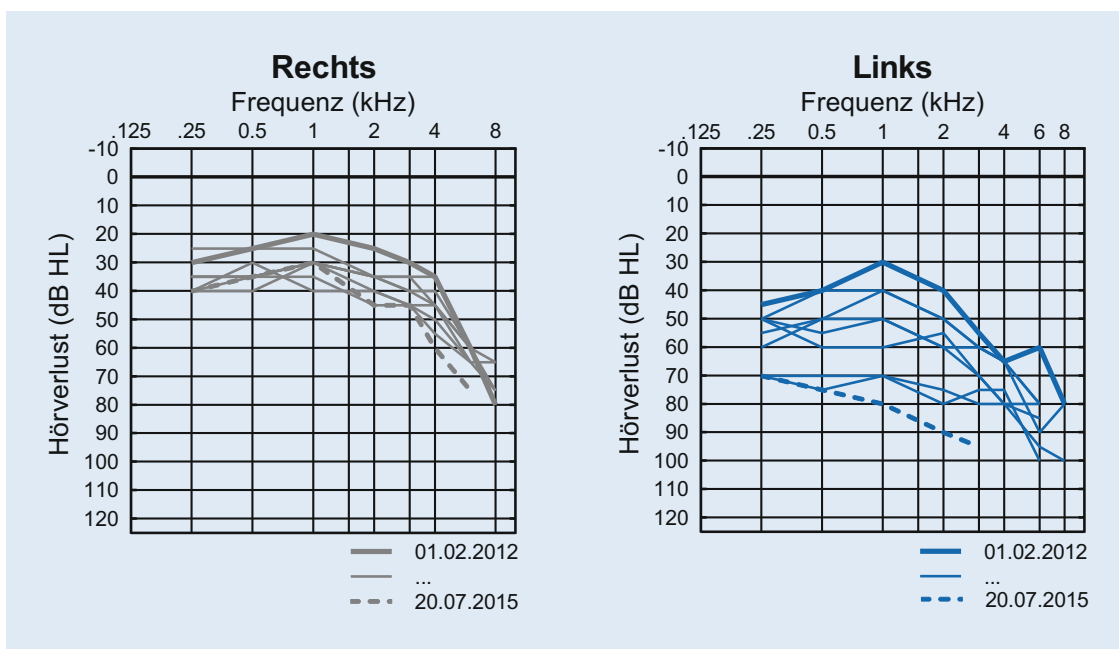

Abb. 2 A Audiometrische Daten zwischen 2012 und 2015; Nachweis der postradiogenen Hörverschlechterung links diotherapie beschrieben wird, kam nicht zur Darstellung (• Abb. 3a) [10]. Zusätzlich zeigte sich eine massive Auftreibung des $\mathrm{N}$. vestibulocochlearis und des $\mathrm{N}$. facialis (• Abb. 3b).

\section{Therapie und Verlauf}

Aufgrund des klinischen und radiologischen Progresses wurde die Indikation zur mikrochirurgischen Tumorentfernung mit neurophysiologischem Monitoring gestellt. Perioperativ erfolgte die i.v.-Gabe des Kalziumantagonisten Nimodipin. Während der Operation über einen retrosigmoidalen Zugang imponierte der $\mathrm{N}$. vestibulocochlearis proximal des eigentlichen Tumors als massiv aufgetrieben (-Abb. 4a). Als Tumorursprung konnte der $\mathrm{N}$. vestibularis inferior identifiziert werden. Intraoperativ kam es zu einer Verschlechterung der akustisch evozierten Potenziale (AEP). Bei bereits hochgradiger Einschränkung des Hörvermögens präoperativ entschloss man sich, den N. vestibulocochlearis zugunsten einer kompletten Tumorentfernung zu resezieren (- Abb. 4 b). Intraoperativ wurde ebenfalls eine kontinuierliche Elektromyographie (EMG) der vom N. facialis innervierten Muskulatur abgeleitet. Hier kam gehäuft zu A-Train-Aktivität, auch ohne Manipulation des Nervs. Als Korrelat zu dieser Spontanaktivität im EMG zeigte sich der N. facialis intraoperativ an der RootExit-Zone ebenfalls massiv aufgetrieben (• Abb. 4c). Ein Gefäß-Nerven-Konflikt als potenzielle Ursache des Hemispasmus facialis konnte nicht identifiziert werden.

Histologisch wurde ein Vestibularisschwannom WHO I mit älteren Blutungen beschrieben. In der histologischen Aufarbeitung des $\mathrm{N}$. vestibulocochlearis fanden sich Zeichen der Demyelinisierung, ödematöse und degenerative Veränderungen mit reduzierter Nervenfaserdichte und Makrophageninfiltraten (• Abb. 5).

Postoperativ kam es zunächst zu einer ausgeprägten fazialen Parese (House \& Brackmann Grad IV), welche sich im Verlauf sehr gut erholte (House \& Brackmann Grad I). Die Hypästhesien V2 und V3 sowie der Hemispasmus facialis zeigten sich komplett regredient. Subjektiv habe sich ebenfalls sich Schwindelsymptomatik gebessert. MRT-Verlaufskontrollen über bis- 

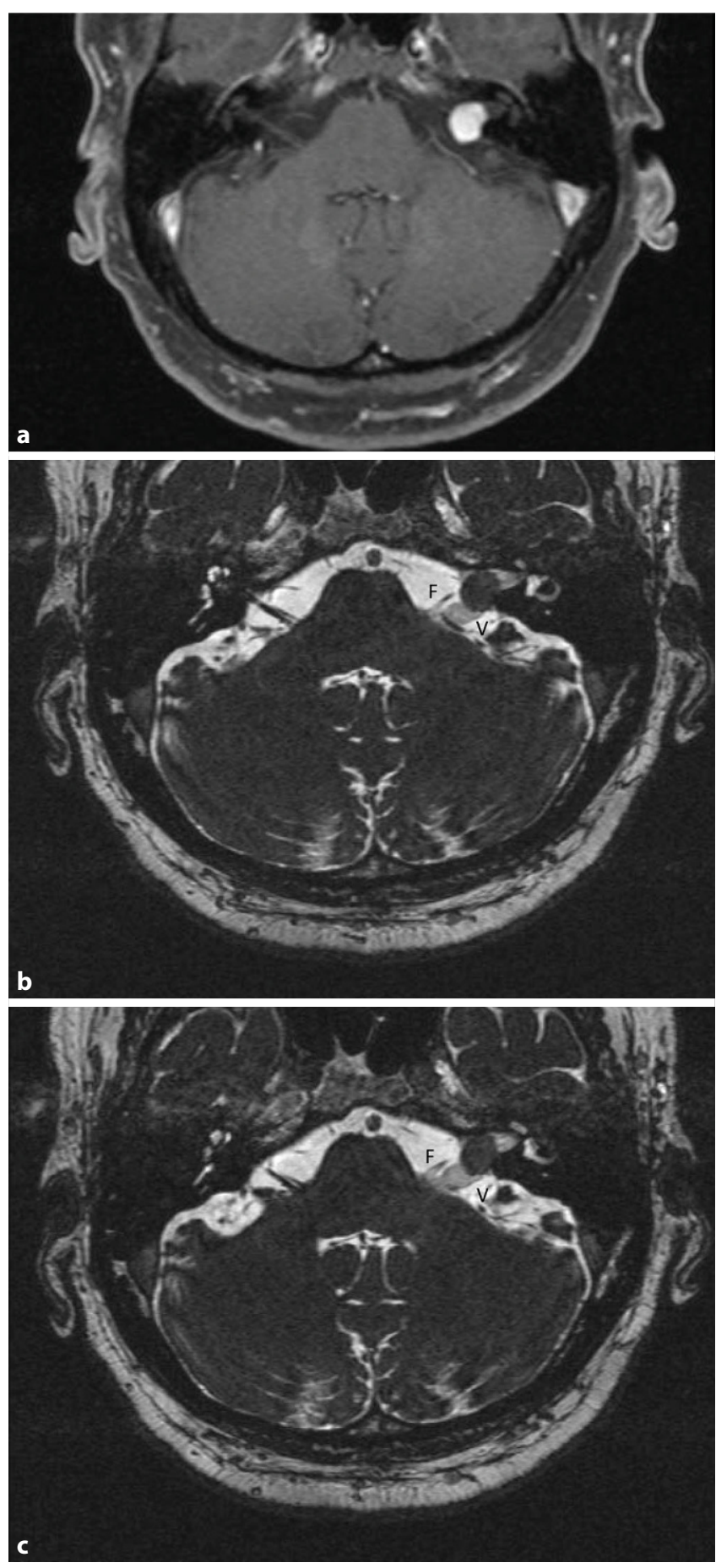

Abb. 3 < cMRT im Jahr 2015. a T1 axial mit Kontrastmittel; Progress der kontrastmittelaufnehmenden Raumforderung im Kleinhirnbrückenwinkel links, Indikation zur Operation; b und c T2 axial; Auftreibung des N.facialis (F) sowie des N. vestibulocochlearis $(\mathrm{V})$

lang 63 Monate bestätigen eine vollständige Tumorresektion (• Abb.6).

\section{Diskussion}

Bei intrameatalen Vestibularisschwannomen stellen radiochirurgische Therapien wie Gamma-Knife oder CyberKnife etablierte Modalitäten dar, welche in den meisten Fällen eine Tumorkontrolle er- linisierung mit chronisch degenerativen Veränderungen des $\mathrm{N}$. vestibulocochlearis. Bereits präoperativ war in der MRTBildgebung auffällig, dass sowohl der $\mathrm{N}$. vestibulocochlearis als auch der N. facialis an Volumen zugenommen haben.

Nach radiochirurgischen Eingriffe sind Komplikationen wie Hypästhesie des N. trigeminus, Hemispasmus facialis oder Hörminderung bereits regelmäßig beschrieben $[2,5]$. Dass diese Effekte auf eine Demyelinisierung durch die vorherige Therapie zurückzuführen sind, konnte bisher jedoch nicht gezeigt werden. Dass Demyelinisierung infolge einer Strahlentherapie auftreten kann und dieser Effekt dosisabhängig ist, konnte bei Tierversuchen gezeigt werden [11].

Bildgebend kam es bei dem gezeigten Fall nach der Gamma-Knife-Therapie zu einem Progress des kontrastmittelaufnehmenden Tumors im Kleinhirnbrückenwinkel. Differenzialdiagnostisch muss neben einem reinen Tumorprogress auch eine maligne Transformation in Betracht gezogen werden, welche in Einzelfällen nach vorheriger Bestrahlung beobachtet werden konnte [7]. Hinweise für Malignität ergaben sich bei unserem Fall nicht.

Neben dem Hörerhalt ist das Vorhandensein und die Ausprägung einer fazialen Parese ein wichtiger postoperativer Aspekt. Typischerweise ist hier das Outcome nach vorheriger Bestrahlung als schlecht einzustufen [4], allerdings konnten wir im vorliegenden Fall postoperativ eine sehr gute Erholung der Parese beobachten. Dies wird möglicherweise durch die perioperative Gabe von Nimodipin begünstigt. Es gibt Hinweise, dass $\mathrm{Ni}$ modipin neuroprotektive Eigenschaften besitzt und einen positiven Einfluss auf die Myelinisierung zu haben scheint [6, 8].

Dieser Fall zeigt erstmals ein morphologisches Korrelat für unerwünschte Effekte nach radiochirurgischer Therapie eines Vestibularisschwannoms und weist darauf hin, dass trotz einer gewissen räumlichen Distanz zum eigentlichen Tumor mit degenerativen Veränderungen nervaler Strukturen zu rechnen sein kann.

Generell empfehlen wir eine Behandlung von Vestibularisschwannomen im Fall eines nachgewiesenen Wachstums, es sei denn die Tumoren sind bei Diagnosestel- 

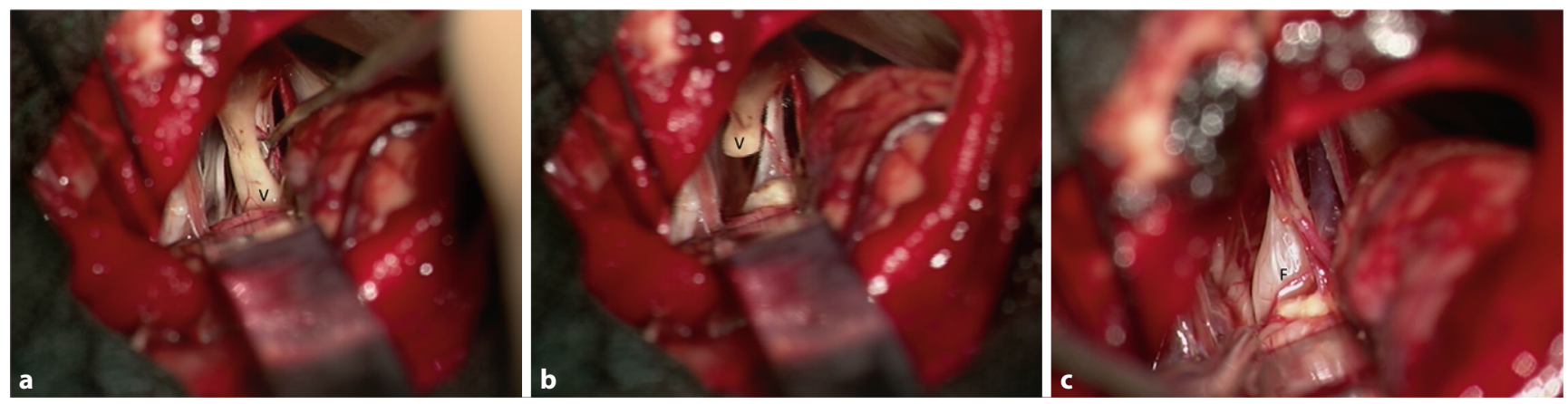

Abb. 4 ム Intraoperative Befunde. a N. vestibulocochlearis mit massiver Auftreibung (V); b Bild nach Resektion des N. vestibulocochlearis; c N. facialis $(F)$ mit massiver Auftreibung an der Root-Exit-Zone
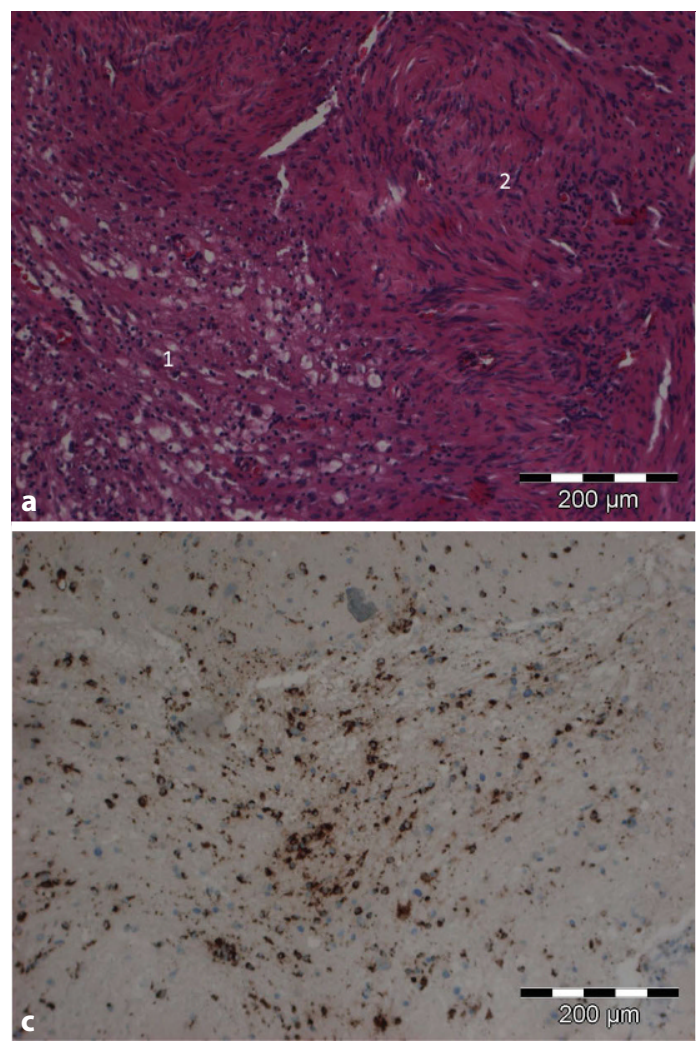
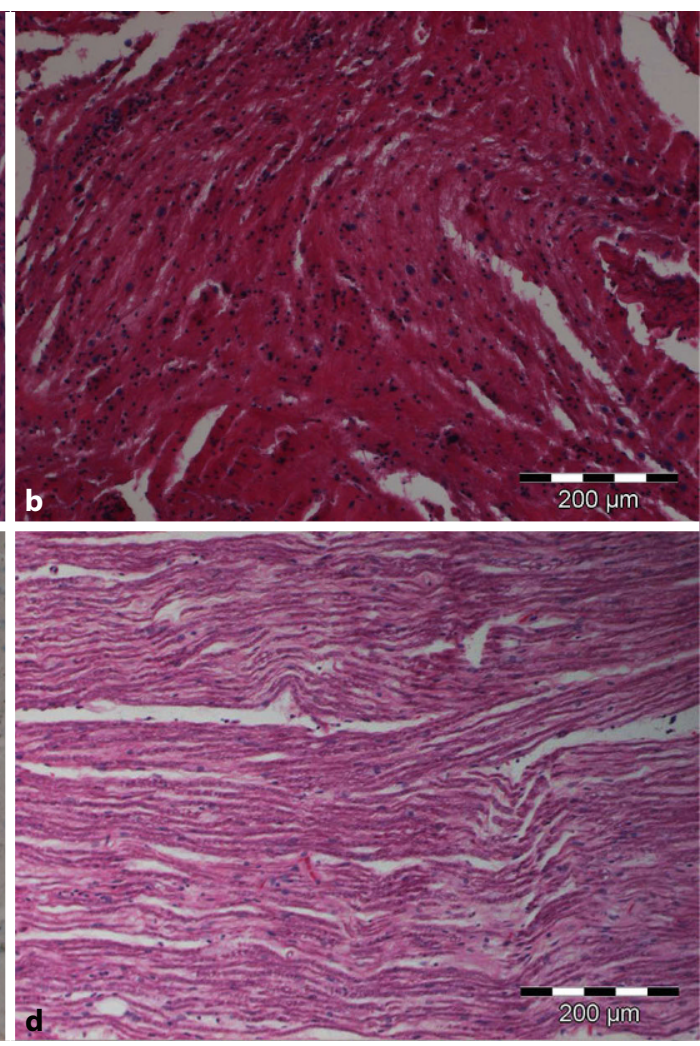

Abb. $5<$ Histologische

Aufarbeitung: N. vestibulocochlearis. a HE: peripheres Nervengewebe mit degenerativen Veränderungen und Zeichen der Demyelinisierung (1), Schwannom (2); b HE: peripheres Nervengewebe mit Ödem und Degeneration; c CD68: Infiltration von Makrophagen im degenerierten peripheren Nervengewebe; d HE: peripheres Nervengewebe des N. vestibulocochlearis eines Gesunden im Vergleich
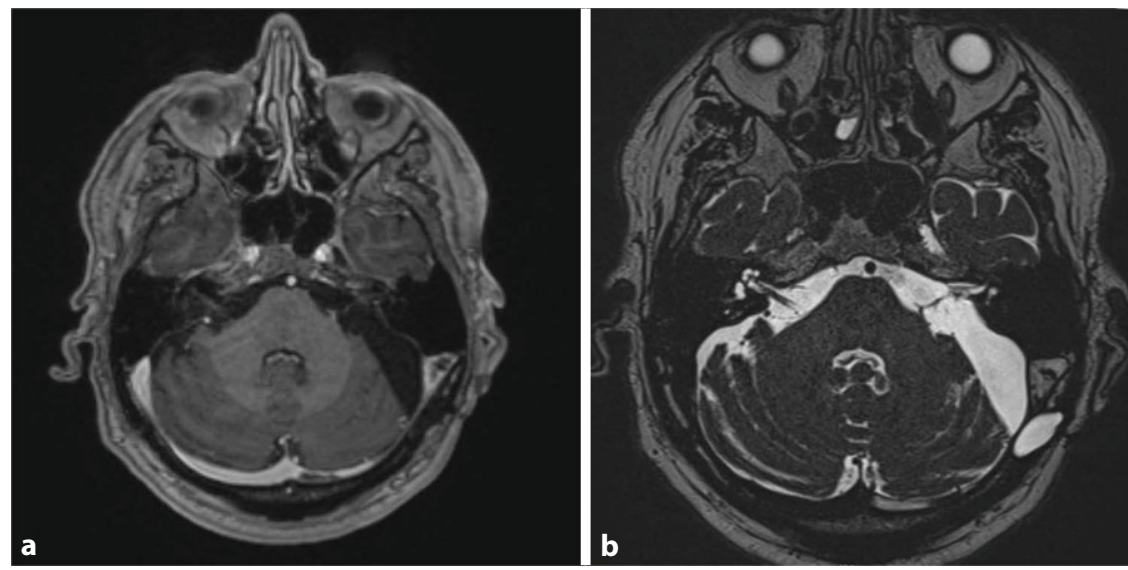

Abb. 6 A cMRT im Jahr 2021. a T1 axial mit Kontrastmittel; kein Anhalt für Rest- oder Rezidivbefund; b T2 axial, Normalisierung des $\mathrm{N}$. facialis lung bereits als Koos 3 oder 4 klassifiziert. In solchen Fällen besteht eine Indikation zur Operation. Bei Tumoren bis Koos 2 ist alternativ eine strahlentherapeutische Behandlung möglich, bei Patienten unter 50 Jahren favorisieren wir auch in diesen Fällen eher den operativen Eingriff, wobei in jedem Fall ein Behandlungskonzept interdisziplinär (HNO, Neurochirurgie und Strahlentherapie) und individuell erstellt werden sollte.

\section{Fazit für die Praxis}

- Radiochirurgische Therapien wie GammaKnife oder CyberKnife sind etablierte Mo- 
dalitäten, welche in den meisten Fällen eine Tumorkontrolle und gute Chancen auf der Hörerhalt erlauben.

- Trotz sorgfältiger Therapieplanung müssen bei der Beratung der Patienten auch seltene Komplikationen berücksichtigt werden.

- Radiochirurgische Eingriffe können zu chronisch degenerativen Veränderungen und einer Demyelinisierung angrenzender neuraler Strukturen führen.

\section{Korrespondenzadresse}

\section{Dr. Maximilian Scheer}

Klinik für Neurochirurgie, Universitätsklinikum Halle, Medizinische Fakultät der Martin-LutherUniversität Halle-Wittenberg

Ernst-Grube-Straße 40, 06120 Halle,

Deutschland

maximilian.scheer@uk-halle.de

Funding. Open Access funding enabled and organized by Projekt DEAL.

\section{Einhaltung ethischer Richtlinien}

Interessenkonflikt. M. Scheer, C. Scheller, J. Prell, C. Mawrin, T. Rahne, C. Strauss und S. Simmermacher geben an, dass kein Interessenkonflikt besteht.

Für diesen Beitrag wurden von den Autoren keine Studien an Menschen oder Tieren durchgeführt. Für die aufgeführten Studien gelten die jeweils dort angegebenen ethischen Richtlinien. Für Bildmaterial oder anderweitige Angaben innerhalb des Manuskripts, über die Patienten zu identifizieren sind, liegt von ihnen und/oder ihren gesetzlichen Vertretern eine schriftliche Einwilligung vor.

Open Access. Dieser Artikel wird unter der Creative Commons Namensnennung 4.0 International Lizenz veröffentlicht, welche die Nutzung, Vervielfältigung, Bearbeitung, Verbreitung und Wiedergabe in jeglichem Medium und Format erlaubt, sofern Sie den/die ursprünglichen Autor(en) und die Quelle ordnungsgemäß nennen, einen Link zur Creative Commons Lizenz beifügen und angeben, ob Änderungen vorgenommen wurden.

Die in diesem Artikel enthaltenen Bilder und sonstiges Drittmaterial unterliegen ebenfalls der genannten Creative Commons Lizenz, sofern sich aus der Abbildungslegende nichts anderes ergibt. Sofern das betreffende Material nicht unter der genannten Creative Commons Lizenz steht und die betreffende Handlung nicht nach gesetzlichen Vorschriften erlaubt ist, ist für die oben aufgeführten Weiterverwendungen des Materials die Einwilligung des jeweiligen Rechteinhabers einzuholen.

Weitere Details zur Lizenz entnehmen Sie bitte der Lizenzinformation auf http://creativecommons.org/ licenses/by/4.0/deed.de.

\section{Macroscopic and microscopic changes of the vestibulocochlear nerve after Gamma Knife treatment}

We report on a case in which macroscopic and microscopic changes of the vestibulocochlear nerve could be observed after radiosurgery of an intrameatal vestibular schwannoma. This case shows for the first time a morphological correlate for undesirable effects after radiosurgical treatment of a vestibular schwannoma and indicates that despite a certain distance to the actual tumor, degenerative changes in neural structures can be expected.

\section{Keywords}

Radiosurgery · Vestibular schwannoma · Complication · Demyelination · Case report

\section{Literatur}

1. Dzierzęcki S, Turek G, Czapski B, Dyttus-CebulokK, Tomasiuk R, Kaczor S, Ząbek M (2020) Gamma knife surgery in the treatment of intracanalicular vestibular schwannomas. Acta Neurol Scand 141(5):415-422. https://doi.org/10.1111/ane. 13220

2. Fu VX, Verheul JB, Beute GN, Leenstra S, Kunst HPM, Mulder JJS, Hanssens PEJ (2018) Retreatment of vestibular schwannoma with Gamma Knife radiosurgery: clinical outcome, tumor control, and review of literature. J Neurosurg 129(1):137-145. https://doi.org/10.3171/2017.3.JNS162033

3. Mahboubi H, Sahyouni R, Moshtaghi O, TadokoroK, Ghavami Y, Ziai K, Lin HW, Djalilian HR (2017) Cyberknife for treatment of vestibular Schwannoma: a meta-analysis. Otolaryngol Head Neck Surg. https://doi.org/10.1177/0194599817695805

4. Rampp S, Strauss C, Scheller C, Rachinger J, Prell J (2013) A-trains for intraoperative monitoring in patients with recurrent vestibular schwannoma. Acta Neurochir 155(12):2273-2279. https:// doi.org/10.1007/s00701-013-1891-x (discussion 2279)

5. Rueß D, Pöhlmann L, Grau S, Hamisch C, Hellerbach A, Treuer H, Kocher M, Ruge MI (2017) Longterm follow-up after stereotactic radiosurgery of intracanalicular acoustic neurinoma. Radiat Oncol. https://doi.org/10.1186/s13014-017-0805-0

6. Scheller C, Wienke A, Wurm F, Simmermacher S, Rampp S, Prell J, Rachinger J, Scheller K, Koman G, Strauss C, Herzfeld E (2014) Neuroprotective efficacy of prophylactic enteral and parenteral nimodipine treatment in vestibular schwannoma surgery: a comparative study. J Neurol Surg A Cent Eur Neurosurg 75(4):251-258. https://doi.org/10. 1055/s-0033-1355164

7. Simmermacher S, Vordermark D, Kegel $T$, Strauss C (2017) Malignisierung eines Vestibularisschwannoms 13 Jahre nach Bestrahlung. HNO 65(9):766-770. https://doi.org/10.1007/ s00106-016-0321-9

8. Tang Y, Zheng X, Ying T, Yuan Y, Li S (2015) Nimodipine-mediated re-myelination after facial nerve crush injury in rats. J Clin Neurosci 22(10):1661-1668. https://doi.org/10.1016/j.jocn. 2015.03.048

9. Windisch PY, Tonn J-C, Fürweger C, Wowra B, Kufeld M, Schichor C, Muacevic A (2019) Clinical results after single-fraction radiosurgery for 1,002 vestibular Schwannomas. Cureus. https://doi.org/ 10.7759/cureus.6390

10. Yang $\mathrm{H}-\mathrm{C}$, Wu C-C, Lee $\mathrm{C}-\mathrm{C}$, Huang $\mathrm{H}-\mathrm{E}$, Lee $\mathrm{W}-\mathrm{K}$, Chung W-Y, WuH-M, GuoW-Y, Wu Y-T, Lu C-F (2020)
Prediction of pseudoprogression and long-term outcome of vestibular Schwannoma after Gamma Knife radiosurgery based on preradiosurgical MR radiomics. Radiother Oncol 155:123-130. https:// doi.org/10.1016/j.radonc.2020.10.041

11. ZhaoZ-F, Yang L-Z, Jiang C-L, Zheng Y-R, Zhang J-W (2010) Gamma Knife irradiation-induced histopathological changes in the trigeminal nerves of rhesus monkeys. J Neurosurg 113(1):39-44. https://doi.org/10.3171/2010.1.jns091116 